\title{
Cole Parameter Estimation from Electrical Bioconductance Spectroscopy Measurements
}

\author{
Fernando Seoane, Member IEEE, Rubén Buendía and Roberto Gil-Pita, Associate Member IEEE
}

\begin{abstract}
Several applications of Electrical Bioimpedance (EBI) make use of Cole parameters as base of their analysis, therefore Cole parameters estimation has become a very common practice within Multifrequency- and EBI spectroscopy. EBI measurements are very often contaminated with the influence of parasitic capacitances, which contributes to cause a hook-alike measurement artifact at high frequencies in the EBI obtained data.

Such measurement artifacts might cause wrong estimations of the Cole parameters, contaminating the whole analysis process and leading to wrong conclusions. In this work, a new approach to estimate the Cole parameters from the real part of the admittance, i.e. the conductance, is presented and its performance is compared with the results produced with the traditional fitting of complex impedance to a depressed semi-circle. The obtained results prove that is feasible to obtain the full Cole equation from only the conductance data and also that the estimation process is safe from the influence capacitive leakage.
\end{abstract}

\section{INTRODUCTION}

Qince the introduction of the Cole function (1) by K.S Cole in 1940, the function and its parameters have been widely used for data representation as well as analysis of spectroscopy and Multi-frequency impedance measurements on Electrical Bioimpedance (EBI) applications, like assessing on tissue contents or tissue status.

From the early applications of EBI spectroscopy (EBIS) to body composition analysis (BCA) already in 1992 [1], the use of EBIS has proliferated to the application areas of tissue characterization like skin cancer detection [2]. EBIS has not only proliferated to other areas but it has deep-rooted into BCA applications, especially through the analysis of the Cole parameters [3]. Therefore Cole parameters estimation from EBI spectral measurements have became a common practice in EBIS applications.

The presence of parasitic capacitances when performing EBI measurements is common $[4,5]$ and may influence notably the obtained EBI data, especially when

Manuscript received March 30th, 2010.

R. Buendia is with the School of Engineering at the University of Borås and the Department of Theory of the Signal and Communications at the University of Alcalá, (e-mail: ruben.buendia@hb.se).

F. Seoane is with the School of Engineering at the University of Borås. Allégatan 1, Borås, Sweden SE-501 90. (tel:+46334354414), email: fernando.seoane@hb.se).

R. Gil-Pita is with the Department of Theory of the Signal and Communications at the University of Alcalá, Ctra Madrid-Barcelona Km 32, Alcalá, Spain ES-28871. (e-mail: roberto.gil@uah.es). using textile-electrodes. A typical measurement artifact caused by the parasitic capacitance is a hook-alike deviation in the EBI spectral data at high frequencies [4]. When an EBI measurement is contaminated with a parasitic capacitance, the measurement will contain a new frequency dispersion that shifts the dominant and intrinsic dispersion of the biological tissue towards the dispersion of the parasitic capacitance. Such deviation in the spectral EBI data influences the Cole parameter estimation process and might mislead the results of the EBI analysis.

Several methods for compensating [3], correcting [6] or minimizing [4] the effect of the afore-mentioned parasitic capacitances on the analysis of EBI data have been reported.

In this work, an original approach for Cole parameter estimation, with the intrinsic benefit of avoiding the influence of the Hook Effect, is presented. The method proposed makes use of the Cole fitting approach recently introduced by Ayllón in [7] applying it on the real part of the Admittance, the conductance.

\section{METHODS}

Test EBI data have been obtained from an experimentally-based Cole model with added measurement noise. In order to extract the Cole parameters, two different Cole fitting methods have been applied on the obtained data, one of the methods based on the conductance and for comparative purposes, a second one based on the complex impedance and semi-circular fitting [8]. The fittings obtained on the impedance plane and Cole parameters estimated from the fittings have been compared with the values originally used to generate the test EBI data.

\section{A. Cole Equation}

In 1940 Cole [9], introduced a mathematical equation that fitted the experimentally obtained EBI measurements (1). This equation is not only commonly used to represent but also to analyze the EBI data. The analysis is based on the four parameters contained in the Cole equation $R_{0}$, $R_{\infty}, \alpha$ and $\tau$, i.e. the inverse of characteristic natural frequency $\omega_{c}$.

$$
Z_{C O L E}(\omega)=R_{\infty}+\frac{R_{0}-R_{\infty}}{1+(j \omega \tau)^{\alpha}}
$$


The value generated by the Cole equation is a complex value containing resistance and reactance. $Z_{C O L E}(\omega)$ is non-linear on the frequency domain and it generates a suppressed semi-circle when plotted in the impedance plane.

\section{B. Cole Equation in Admittance}

Since the admittance is the mathematically inverse of the impedance $Y=Z^{l}$, inverting (1) and equalizing $Y_{0}$ with $\left(R_{0}\right)^{-1}$ and $Y_{\infty}$ with $\left(R_{\infty}\right)^{-1}$ the Cole function in admittance form $Y_{C O L E}(\omega)$ is obtained (2).

$$
Y_{C O L E}(\omega)=Y_{0}+\frac{Y_{\infty}-Y_{0}}{1+\frac{Y_{\infty}}{Y_{0}}\left(j \frac{\omega}{\omega_{c}}\right)^{-\alpha}}
$$

Applying $j^{\alpha}=\cos (\alpha \pi / 2)+j \sin (\alpha \pi / 2)$ being $j=(-1)^{1 / 2}$ on (2) it is possible to decompose $Y_{C O L E}(\omega)$ in its real and imaginary parts obtaining the conductance $G(\omega)$ in (3).

$$
G_{C O L E}(\omega)=Y_{0}+\frac{\left(Y_{\infty}-Y_{0}\right)\left(1+\frac{Y_{\infty}}{Y_{0}}(\omega \tau)^{-\alpha} \cos (\alpha \pi / 2)\right)}{1+\frac{Y_{\infty}^{2}}{Y_{0}^{2}}(\omega \tau)^{-2 \alpha}+2 \frac{Y_{\infty}}{Y_{0}}(\omega \tau)^{-\alpha} \cos (\alpha \pi / 2)}
$$

The equation given in (3) can be used to fit the real part of the inverse of the impedance generated and this way the Cole parameters can be estimated in an analogous manner to [7].

\section{Non-Linear Least Squares Fitting}

This method obtains the best coefficients for a given model that fits the curve, the method aims at minimizing the summed squared of the error between the data point and the fitted model (4).

$$
\min \sum_{i=1}^{N} e_{i}^{2}=\min \sum_{i=1}^{N}\left(y_{i}-\bar{y}_{i}\right)^{2}
$$

Where $\mathrm{N}$ is the number of data points included in the fitting.

This method is implemented in Matlab $®$ directly with the function fit and the option Non Linear Least Squares that fits the generated data as to a non-linear real parametric model with coefficients, using the natural frequency $\omega$ as independent variable. In our case the model used is $G_{C O L E}(\omega)$ given in (3) and the model coefficients are $Y_{0}, Y_{\infty}, \tau$ and $\alpha$.

\section{Noise model and Data Generation}

To simulate the data, a Cole function with Cole parameters extracted from a wrist-to-ankle 4-electrode EBI experimental measurement has been implemented. The values used for the Cole parameters are as follows: $R_{0}=750, R_{\infty}=560, \alpha=0.68$ and $\tau=3.55 \times 10^{-6}$. EBI data

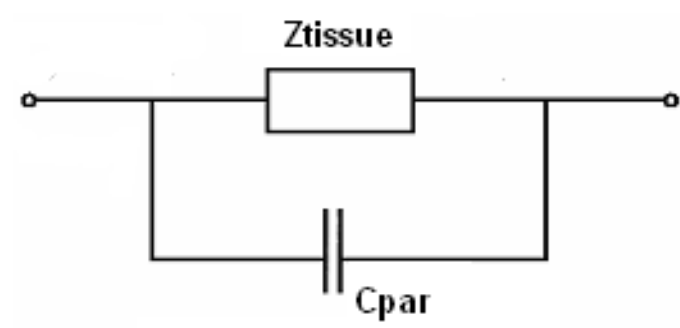

Fig. 1 Ztissue Cole based model in parallel with a parasitic capacitance. have been generated with and without parasitic capacitance, $C_{\text {par }}$, simulating the presence of a parasitic capacitance as shown in Fig. 1.

The 100 impedance spectra have been created with values of frequency spaced logarithmically as suggested in [3].

From 100 wrist-to-ankle measurements of complex EBI, measurement noise has been characterized obtaining the mean, Standard Deviation and the spectral components. The EBI measurements were performed with the 4-electrode method using the SFB7 impedance spectrometer manufactured by Impedimed. Using the characteristics of the measurement noise, synthetic noise has been generated and added to the simulated EBI data.

\section{E. Cole Parameter Estimation Performance Analysis}

The performance of the two applied methods has been assessed by studying the Mean Absolute Percentage Error (MAPE) produced in the estimation of each Cole parameters, as shown in (5).

$M A P E=\frac{\sum_{n=1}^{N}\left|\frac{x-\bar{x}}{\bar{x}}\right|}{N} * 100$

Where $x$ represent the estimated value of the Cole parameter under study i.e. $R o, R_{\infty}, \alpha, f c$ and $\bar{x}$ the original value of the parameter under study. $N$ is the total number of estimations.

\section{RESUltS}

\section{A. EBI Data Fitting}

Fig. 2 contains three different impedance plots. In Fig 1.A) the resistance spectrum is plotted, and it is possible to see the agreement of all in one trace, the crossed trace is representing the real part of the Cole function fitted by the impedance plane method for a impedance contaminated with a capacitance in parallel.

In Fig. 1.B) and 1.C), reactance spectrum and impedance plot respectively, it is possible to observe a remarkable deviation on both traces representing the impedance contaminated with $15 \mathrm{pF}$ and the curve fitted on the impedance plane, solid thick and crossed trace respectively.

In all three plots contained in Fig. 1 the fit done on the 
A) Resistance Spectrum

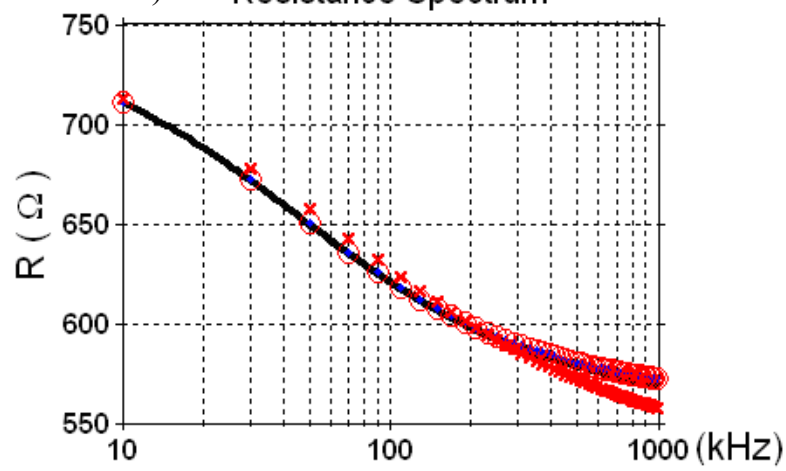

B) Reactance Spectrum
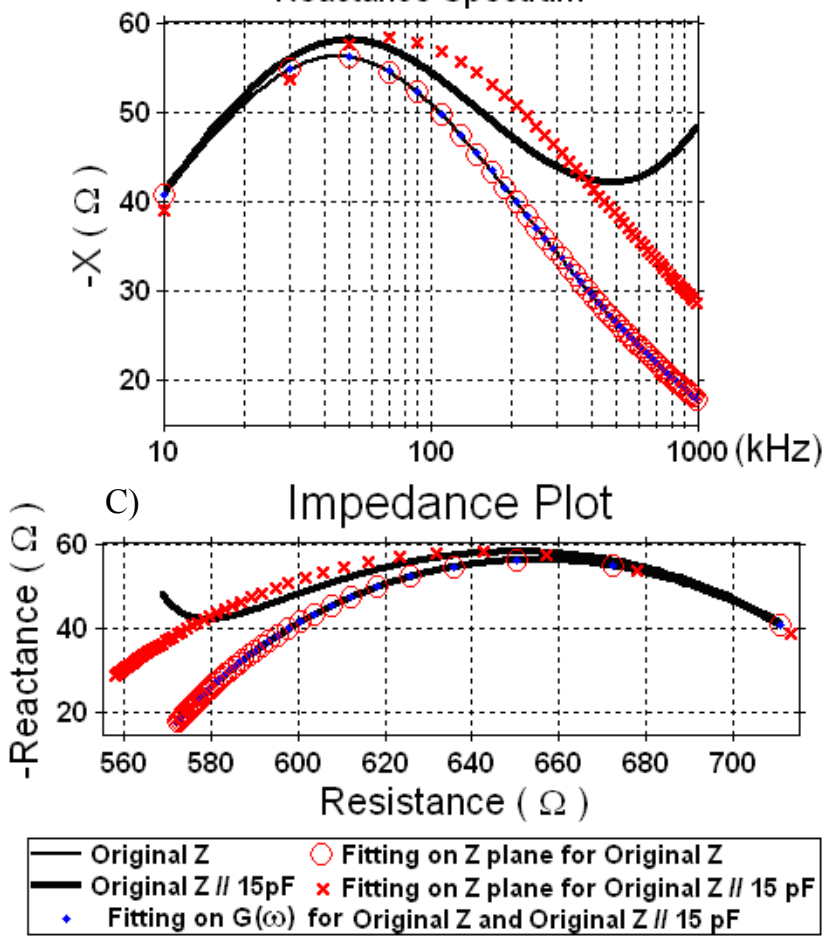

Fig. 2. Graphs showing the generated EBI data and the results from the performed curve fittings on the impedance plane and the conductance domain. Resistance spectrum plotted in A), Reactance Spectrum in B) and the impedance plot on C).

conductance, dotted trace, matches perfectly the values of the original impedance without parasitic capacitance, plotted with solid thin trace. Note that since the fitting obtained from $\mathrm{G}(\omega)$ produces the same values independently of the presence of parasitic capacitance, there is only one trace indicating both fittings produced from $\mathrm{G}(\omega)$.

The fitting done on the impedance plane, produces a perfect match with the original impedance data, represented with circular markers and thin solid line respectively.

\section{B. Cole Parameters}

The MAPE obtained from both fitting approaches for each of the estimated Cole parameters is listed in Table I. The parameters have been estimated from synthetic EBI
TABLE I. MAPE OBTAINED FROM BOTH APPROACHES FOR ALL FOUR COLE PARAMETERS

\begin{tabular}{c|c|c|c|c}
\hline$(\%)$ & Ro & Rinf & $f_{c}$ & Alpha \\
\hline G $(\omega)$ fitting & 0.04 & 0.09 & 0.72 & 0.04 \\
\hline Zfitting // 0pF & 0.03 & 0.09 & 0.48 & 0.30 \\
\hline Zfitting // 5pF & 0.29 & 1.51 & 13.73 & 3.85 \\
\hline Zfitting // 10pF & 0.55 & 3.31 & 31.57 & 7.62 \\
\hline Zfitting // 15pF & 0.69 & 5.24 & 54.98 & 10.40 \\
\hline
\end{tabular}

data containing different values of parasitic capacitance, ranging from $0 \mathrm{pF}$ to $15 \mathrm{pF}$.

Since the fitting in the conductance plane, generates the same EBI fitted data independently on the value of $C_{\text {par }}$, the obtained MAPE is the same and consequently only one row is used to report the produced MAPE.

In Table I it is possible to observe that for the case of $C_{p a r}=0$ both estimation approaches produce MAPE values in the estimation of the Cole parameters that are extremely low.

The influence of $C_{p a r}$ on the estimation method based in the fitting on the impedance can be appreciated also in the obtained MAPE values.

Fig. 3 shows that both fitting methods provide very good estimations for the Cole parameters when there is no $C_{p a r}$, and the impedance plane fitting method provide a not accurate estimation for $C_{\text {par }}$ in parallel of $15 \mathrm{pF}$.

Fig. 3 shows the actual values for the Cole parameters and the corresponding estimated values from conductance domain and the impedance plane for EBI data free of parasitic capacitance and contaminated with $15 \mathrm{pF}$. In both methods, all estimations done on data free of parasitic capacitive effect produce a very accurate
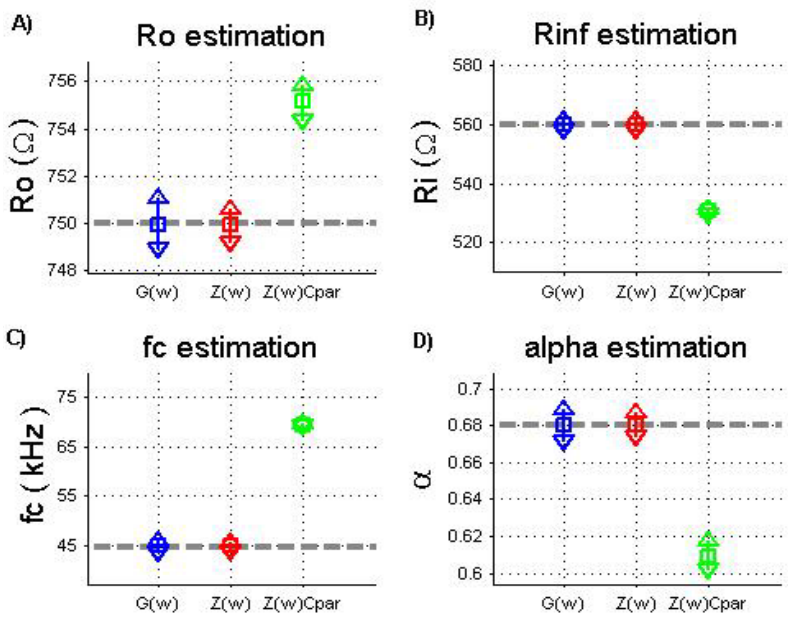

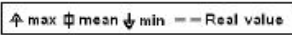

Fig.3. The estimated Cole parameters from both approaches for EBI data free of parasitic capacitance and EBI data contaminated with a Cpar of $15 \mathrm{pF}$. 
estimation. Again, in all 4 graphs contained in Fig. 3, it is possible to appreciate that the estimation done on the impedance plane for EBI data with parasitic capacitive effect present produces highly biased values.

\section{DISCUSSION}

The results indicate that for immitance data without any capacitive parasitic influence both fittings methods performed very well producing a very accurate estimation of the parameters. This is expected since the EBI data presents a single predominant dispersion like the Cole function. Unfortunately obtaining artifacts free immitance measurements is very unrealistic.

Once the data contain any deviation caused by a parasitic capacitance the performance of the fitting done on the impedance plane begins to worsen producing wrong estimations of the Cole parameters. This is due to the fact that this type of fitting tries to fit data containing 2 predominant dispersions into a single dispersion model. The error in the estimation of $R_{0}$ is smaller because at low frequencies the influence of the parasitic capacitance is hardly noticeable.

On the other hand the fittings and the estimation of the Cole parameters from the conductance data is not slightly influenced by the presence of a parasitic capacitance. This was expected since the parasitic capacitance will modify the admittance by adding its value to the imaginary part of the admittance, the subsceptance, without modifying the real part of the admittance. This leaves a conductance with a single predominant dispersion being fitted to the real part of the $Y_{C O L E}$.

\section{CONCLUSION}

This paper shows that it is possible to estimate the Cole parameters accurately from the conductive part of the admittance without the need to measure the imaginary part of the electrical bio-admittance. This approach brings the same advantages to the immitance measurement process than the resistance-based Cole parameter estimation presented in [7], but it also benefits from an intrinsic mechanism to avoid the influence of the Hook Effect.

This approach suggests that in order to estimate the Cole parameters it is only necessary to measure the electrical conductance of a biological system. Any application of EBI measurements that performs Cole model parameters estimation as based of its data analysis e.g. body composition assessment for nutritional status would benefit from novel approach presented here. Of course this can be only applicable in the range of the $\beta$ dispersion and further studies with experimental data must be done to find other limits of its applicability.

\section{REFERENCES}

[1] J. R. Matthie, P. O. Withers, M. D. Van Loan et al., "Development of a commercial complex bio-impedance spectroscopic (CBIS) system for determining intracellular water (ICW) and extracellular water (ECW) volumes." pp. 203-205.

[2] P. Aberg, I. Nicander, J. Hansson et al., "Skin cancer identification using multifrequency electrical impedance - A potential screening tool," IEEE Trans. Bio. Med. Eng., vol. 51, no. 12, pp. 20972102, 2004.

[3] A. De Lorenzo, A. Andreoli, J. Matthie et al., "Predicting body cell mass with bioimpedance by using theoretical methods: a technological review," J Appl Physiol, vol. 82, no. 5, pp. 1542-58, May, 1997.

[4] H. Scharfetter, P. Hartinger, H. Hinghofer-Szalkay et al., "A model of artefacts produced by stray capacitance during whole body or segmental bioimpedance spectroscopy," Physiological Measurement, vol. 19, no. 2, pp. 247-261, 1998.

[5] P. Mirtaheri, S. Grimnes, Ø. G. Martinsen et al., " A new biomedical sensor for measuring PCO2," Physiol. Meas., vol. 25,

pp. $421 \quad 436,2004$.

[6] R. Buendia, F. Seoane, and R. Gil-Pita, “ Novel Approach for Removing the Hook Effect Artefact from Electrical Bioimpedance Spectroscopy Measurements," ournal of Physics: Conference Series, 2010.

[7] D. Ayllón, F. Seoane, and R. Gil-Pita, "Cole Equation and Parameter Estimation from Electrical Bioimpedance Spectroscopy Measurements - A Comparative Study," in Engineering in Medicine and Biology Society, 2009. EMBC 2009. Annual International Conference of the IEEE Minneapolis, 2009, pp. 3779-3782.

[8] B. H. Cornish, B. J. Thomas, and L. C. Ward, "Improved prediction of extracellular and total body water using impedance loci generated by multiple frequency bioelectrical impedance analysis," Phys. Med. Biol., vol. 38, no. 3, pp. 337-346, 1993.

[9] K. S. Cole, "Permeability and impermeability of cell membranes for ions.," Quant. Biol., vol. 8, pp. 110-122, 1940. 